\title{
DIGITAL IMAGING
}

\author{
Hylton B Meire, Ara Darzi, Nicholas Lee
}

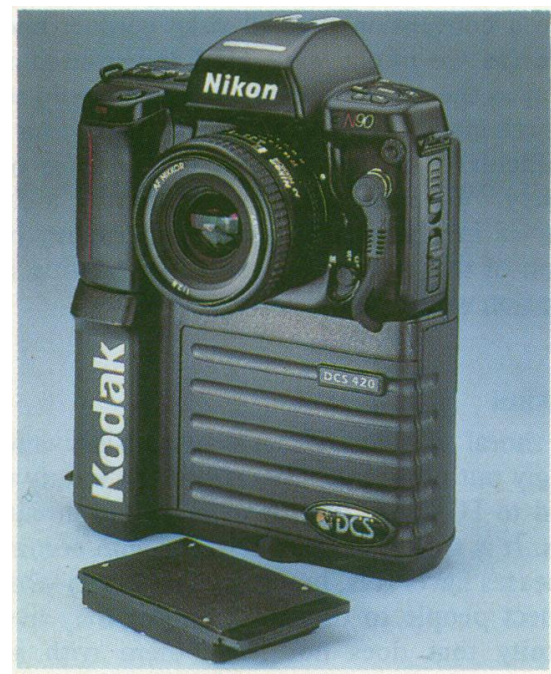

Kodak digital camera system based on Nikon F90 camera.

\section{Digital radiology}

\section{Types of digital radiological imaging}

- Nuclear medicine

- Ultrasound

- Computed tomography

- Digital vascular imaging and digital subtraction angiograms

- Magnetic resonance imaging

- Digital fluorography

- Computed radiography
Pictures have been traditionally taken with photographic film, which then has to be processed before they can be viewed. The development of digital cameras now allows pictures to be taken and printed almost immediately. In medicine this can be an important advantage, and, even though the quality of digital images is currently lower than that of photographic film, the huge advantage of immediate images often far outweighs the slight loss of resolution. Digital cameras are now available to replace the standard $35 \mathrm{~mm}$ camera for taking routine clinical photographs as well as for use in specialised areas of medicine.

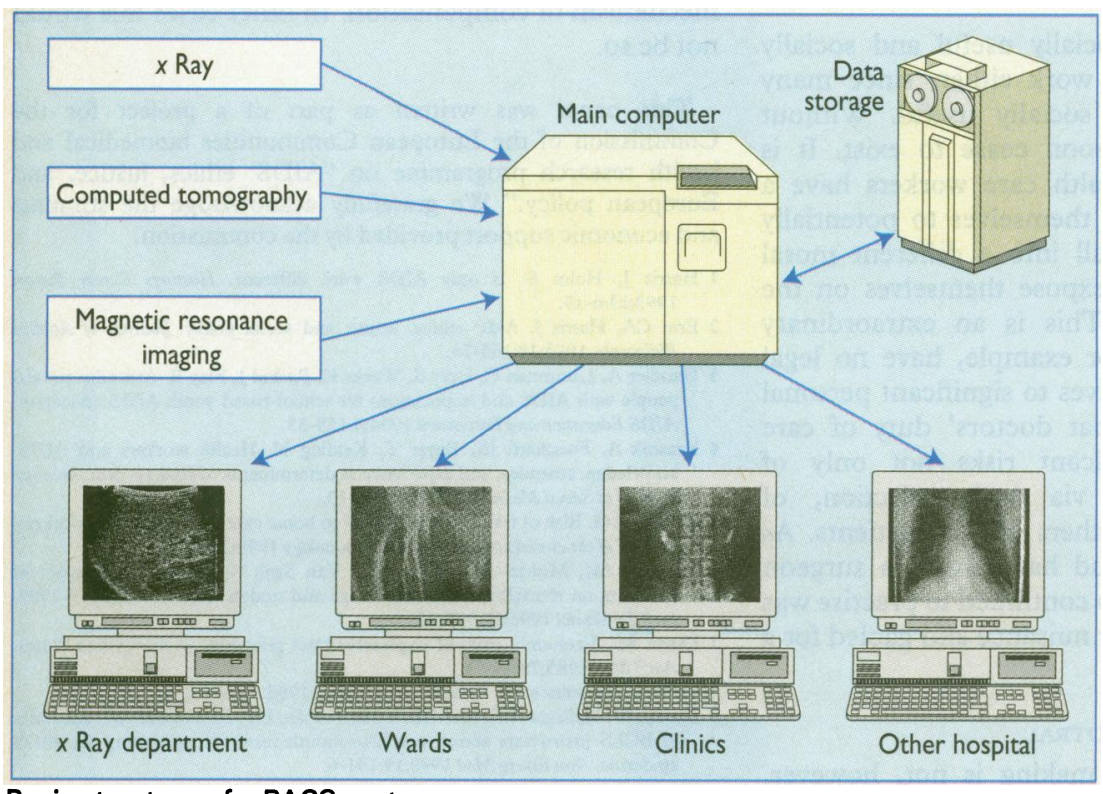

Basic structure of a PACS system.
Photographic film has served as the reception, storage, and display medium for $x$ ray images for the past 100 years. Indeed, $x$ rays were discovered because of their effect on photographic emulsion. When ultrasound scanning was developed the images were displayed on a monitor and were transitory. At that time digital storage was in its infancy, and it was impossible to store large numbers of images. Photographic film was cheap and readily available and so was chosen to provide a permanent record of the investigation.
However, with the current expansion in the variety of digitally acquired images and the increasing capacity of digital storage systems, the possibility of dispensing with photographic film has now arisen. Filmless imaging can lead to important savings in disposable resources and staff time and a major improvement in departmental efficiency.

\section{Picture archiving and communication system}

Storage of digital images is undertaken by a picture archiving and communication system (PACS). This term is now accepted jargon but, like all jargon, is becoming misused. Each component of PACS is fundamental to the success of the system. Pictures must be captured, stored, retrieved, distributed, and displayed at multiple sites for a system to be worthy of the name. 


\begin{tabular}{ll}
\hline Storage capacity needed to hold digital images \\
Digital image & Size of image file \\
Ultrasound scan & $250 \mathrm{~kb}$ \\
Computed tomogram or magnetic resonance & \\
image & $1 \mathrm{Mb}$ \\
Digital mammogram & $4 \mathrm{Mb}$ \\
High resolution chest $x$ ray & $16 \mathrm{Mb}$ \\
One complete computed tomography or & \\
magnetic resonance imaging examination & $100 \mathrm{Mb}$ \\
One week's digital images (uncompressed) & $100000 \mathrm{Mb}$ (100 gigabytes) \\
One year's digital images (uncompressed) & $5000000 \mathrm{Mb}$ (5 terabytes) \\
One high density floppy disk & $1.4 \mathrm{Mb}$ \\
Typical desk top PC hard disk & $250 \mathrm{Mb}$ \\
\hline
\end{tabular}

Although this may seem simple, there are serious problems to be overcome. Almost all radiology systems produce their images in different formats, and the digital image files are often very large. Fortunately, the capacity of data storage systems is increasing at the rate of one order of magnitude every five years, and their costs are steadily reducing. However, the capacity required for a major radiology department to hold all its images on line is still technologically and financially unachievable.

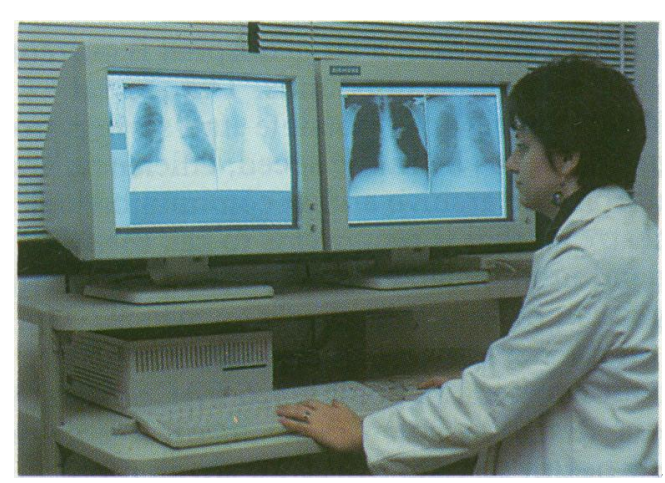

Workstation using two display screens for all types of radiological examinations.

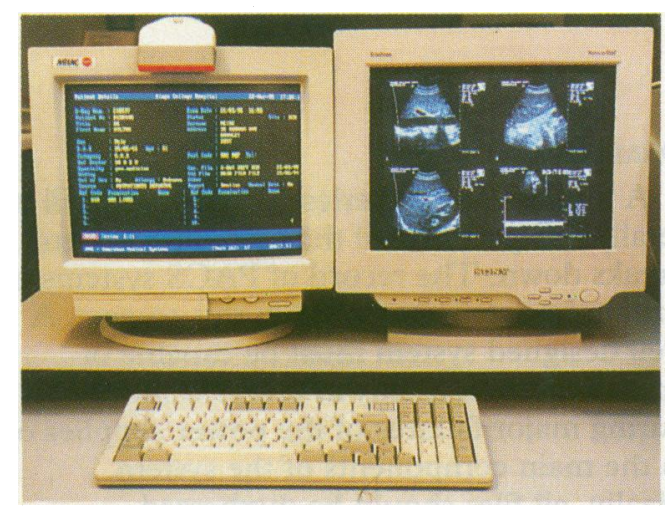

Workstation for a PACS system for ultrasound scans only, with one display screen and a patient data screen.

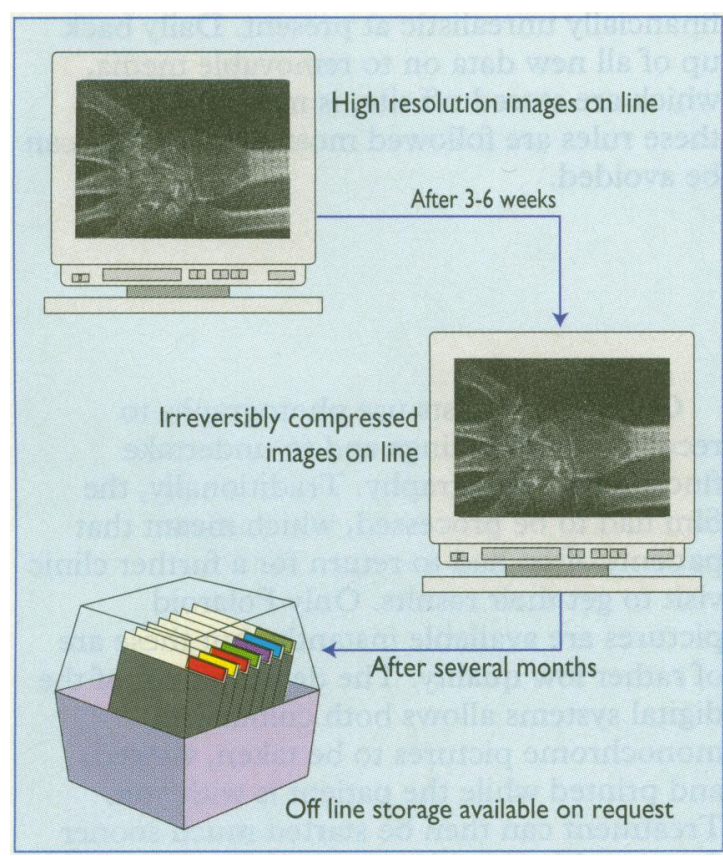

Sequence of image compression and storage.
The display systems present a further obstacle. Radiologists are used to having eight or more viewing boxes of films on display at once, often with a mixture of examinations from one patient under review simultaneously. To reproduce this with large, high resolution computer screens and associated software is very expensive. Most practical systems use only two or four screens at each workstation. In addition the handling of electronic images requires very different disciplines from the radiologist to those he is used to with film.

\section{Realistic systems}

If a usable and cost effective system is to be implemented it is necessary to make a few compromises. The commonest options are:

Store only certain examinations (such as ultrasound, computed tomographic, or magnetic resonance scans). This has the advantage that the radiologist can get used to handling electronic images before being subjected to a totally digital environment, but the economies associated with lack of film will not be fully realised.

Hold images on line for a limited period only-This option is satisfactory if very few patients return for follow up examinations, but it can be a serious problem in a centre with many long term patients; exactly the type of institution that might benefit from PACS.

Compress image data files - This option is always practised to some degree. With modern software it is possible to compress an average radiology image file by about 2.5:1 with no loss of detail. Greater compression results in loss of data, so called "lossy" or "irreversible" compression. However, compressions of 6:1 are almost undetectable in the reconstructed image, and it has been claimed that a chest $x$ ray can be compressed 25:1 before diagnostic information is lost.

In practice at least two of these options are followed in most digital departments to date. Most practical and effective PACS systems store all the department's images and have a "hot file" of rapidly accessible, "losslessly" compressed images that are less than a few weeks old. When an image has not been accessed for a period in excess of this it is irreversibly compressed and passed to the main archive. It can be retrieved from here more slowly. After a further period of inactivity, typically several months, images may be passed to an offline storage device and can be retrieved only when notice has been given in advance.

\section{Integration with management systems}

Most large radiology departments now use computerised management systems. These systems hold all the details of patients' radiology history, appointments, reports, etc, and include a master index. If a PACS is added to such a system it is essential that the two are fully integrated. Surprisingly, this is often not done, and thus one may have to search for the images and the relevant report on two separate systems. Any new PACS system must include a fully functional departmental management system. 


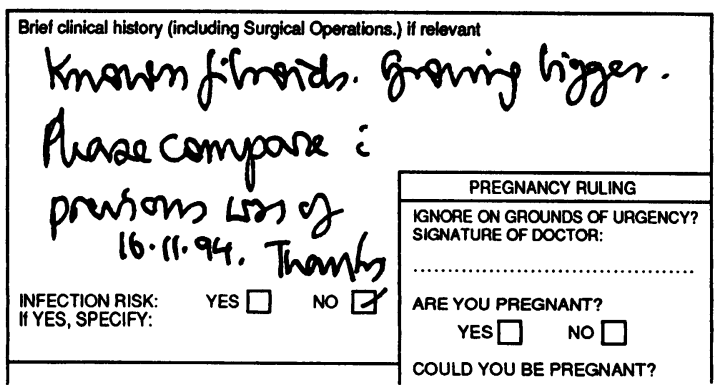

Part of a hand written request form that had been scanned into a computer, compressed, and stored. It is then available for printing or display when required.
Advantages of filmless imaging
- No film handling
- No film processing
- No enveloping of films
- No filing of film envelopes
- No retrieving of film envelopes
- No searching for lost films
- No sending films to wards or clinics
- Images rapidly available
- Greater efficiency
- Teleradiology

Paperwork-There is no logic in having a filmless department if paper request forms and reports still have to be handled and filed. Very few PACS systems address this problem. A fully functional radiology management and PACS system must include the facility for direct electronic requesting of examinations and should be able to convert paper requests into digital form for storage on the system. Only when this is achieved are the full advantages of a filmless and paperless department likely to be realised.

\section{Is PACS cost effective?}

The capital cost of a full PACS system in a large hospital is currently likely to be of the order of tens of millions of pounds. The annual maintenance costs are likely to outweigh any possible staff savings from being filmless. The main gain will thus be in the speed, efficiency, and reliability of image retrieval from the archive and in communication. If a surgeon wants films in theatre all that is required is to call them up on the console, far quicker and more reliable than current $x$ ray storage and retrieval services. Images can be available almost anywhere, even at a remote hospital if it is on the network (teleradiology). Computer technology has now just about reached the point when PACS becomes a realistic prospect for anyone designing a new department. It is not a cheap option, but in the new health service, where we are all more conscious of efficiency, it can allow us to offer a better service to our patients and colleagues.

$\begin{array}{ll}\text { Suppliers of digital imaging systems } \\ \text { Company } & \text { Product } \\ \text { Kodak } & \text { Digital camera system } \\ \text { Occulab } & \text { Digital fundus camera } \\ \text { ImageNet } & \text { Digital fundus camera } \\ \text { Philips } & \text { PACS system } \\ \text { Siemens } & \text { PACS system } \\ \text { SIMIS } & \text { PACS system } \\ \text { AMS } & \text { PACS system }\end{array}$

\section{Data security}

A filmless and paperless department will be totally incapacitated if the computer system breaks down. The record of PACS systems in Britain to date is not good in this respect. A well designed system must be capable of operating, even if only in crippled mode, during major failures of the power supplies or in the main components of the system. Ideally, all files should be duplicated, preferably by all the hard disks in the computer being duplicated, so called disk mirroring. This is technically possible but is financially unrealistic at present. Daily back up of all new data on to removable media, which are stored off site, is mandatory. If these rules are followed most catastrophes can be avoided.

\section{Digital ophthalmic photography}

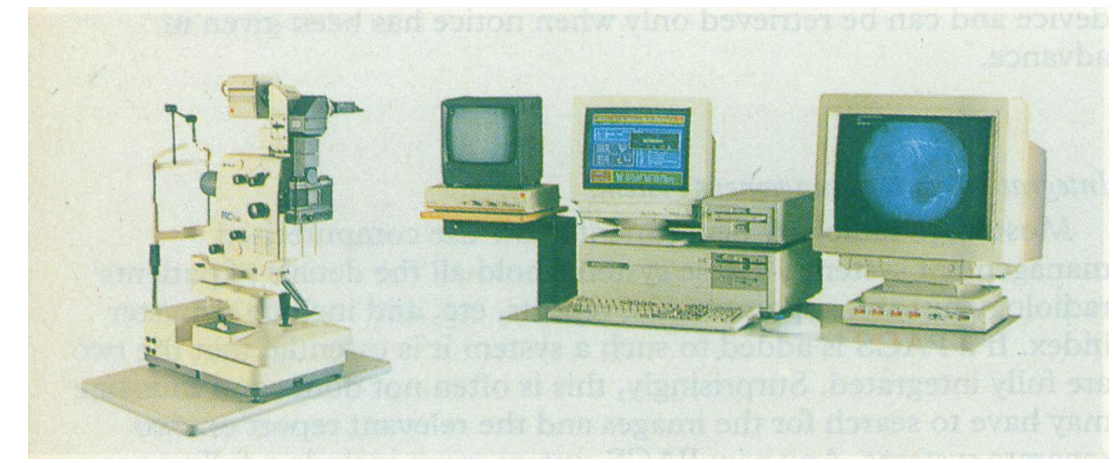

Computer based system for capturing and analysing fundus images.

Ophthalmologists use photographs to record retinal findings and to undertake fluorescein angiography. Traditionally, the film had to be processed, which meant that patients often had to return for a further clinic visit to get their results. Only Polaroid pictures are available instantly, but these are of rather low quality. The development of the digital systems allows both colour and monochrome pictures to be taken, viewed, and printed while the patient is with you. Treatment can then be started much sooner than would otherwise be possible. 


\section{Virtual reality}

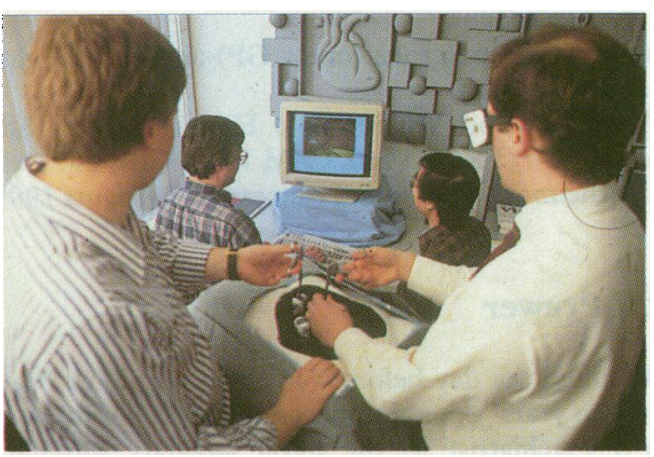

Practising an operation with virtual reality simulation.

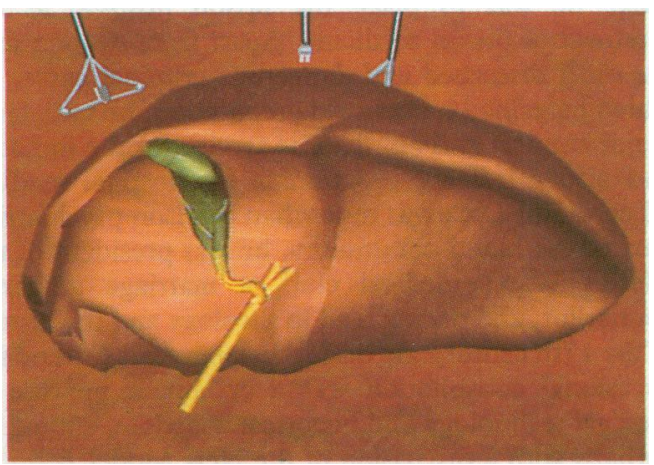

Screen image of a virtual liver.

The picture of practising an operation with virtual reality simulation is reproduced from J Coleman et al, Brf Surg 1994;81:1709-11, with permission of Blackwell Science. The picture of a virtual liver is reproduced with permission of Ciné-Med, Woodbury, Connecticut, USA.

Hylton B Meire is consultant radiologist, Kings College Hospital, London; Ara Darzi is consultant surgeon, minimal access surgery unit, St Mary's Hospital, London; and Nicholas Lee is consultant ophthalmologist, The Western Eye and Hillingdon Hospitals.

The ABC of Medical Computing is edited by Nicholas Lee (nicklee@online.nildram.co.uk) and Andrew

Millman, occupational physician, Gloucestershire Royal Hospital (af89@dial.pipex.com).

The ABC of Medical Computing is now available on the Internet (home page

http://www.cityscape.co.uk/users/dl88/abcmc/abcmc.htm)
Virtual reality refers to a computer generated representation of an environment that allows sensory interaction, thus giving you the impression that you are actually present. The immense complexity of human anatomy and the huge computational power needed to simulate it have so far limited the practical application of virtual reality technology in medicine. This technology has been most closely associated with the entertainment industry in the form of virtual reality video games.

The three ways of experiencing a virtual environment are through sound, sight, and touch. Virtual reality systems create a stereoscopic "visual immersion" by generating two slightly differing images that are alternately presented to each eye, giving the impression of a three dimensional image. This is usually achieved by positioning two small liquid crystal display (LCD) screens in front of each eye-a head mounted display.

\section{Medical applications}

The simulation of realistic tactile and force feedback has been a major obstacle to the application of virtual reality systems in medicine. Currently virtual reality systems are being used in preoperative planning and training in minimally invasive surgery and have the potential to become powerful tools for teaching anatomy.

Computer generated simulators have been developed that allow images to be imported from computed tomography or magnetic resonance imaging. These permit an unparalleled view of anatomic structures previously accessible only by dissecting a cadaver. The advantage of this system is that it allows surgeons to plan and rehearse complex operations - such as reconstructive surgery or neurosurgerybefore the patients ever reach theatre.

Minimally invasive surgery-Training in laparoscopic surgery has been under scrutiny after reports of serious complications. At present, initial training involves manipulating inanimate objects in purpose built training boxes. These allow the development of hand-eye coordination but lack anatomical detail and are therefore of limited use. A virtual reality simulator of laparoscopic cholecystectomy has been developed that allows a surgeon to practice manipulation of instruments and organ without compromising a patient's safety. The system resembles a conventional simulator but presents an anatomically correct image of the biliary system and allows organs to be grasped, retracted, and cut. The system is still at the prototype stage, but it has great potential for use in teaching and evaluating trainee surgeons. Another use for this system is in the design of new instruments before the manufacture of prototypes.

\section{The importance of friendship}

She was my oldest friend, my first real friend. We met aged $3^{1 / 2}$ at nursery school. I remember that I had a tail made of string and we played a game of cats and mice. We lived less than a mile apart, went to the same schools, and for the next nine years or so we were best friends. Most of our time was spent together, both in school and out. Then my family moved house and although we kept in touch we did not see each other often.

We went to different medical schools and I qualified two years before she did, since she had taken time to work on a kibbutz, where she met the man she later married. She had her first child and I went to see her. I was a senior house officer on a busy one in three rota and she was a full time wife and mother-but there was still a strong bond from our childhood days and it seemed as though we could pick up without a break. We were no different as adults than we had been as children. She had a second child and we lost contact, although I used to look her up in the Register from time to time to see where she was. Then 18 months ago, myself now married and settled in a consultant post, I wrote to her and we spoke on the telephone. Her marriage had broken up and she was a general practitioner bringing up her two children alone. I said that I would go and see her, but I was pregnant with my second child, and somehow I just kept putting it off. There never seemed to be time.

Tonight her elder brother telephoned me. She died of ovarian cancer two days ago aged 39. A private person, it seemed that she had not wanted people to know that she was ill. We had not really been close in adult life but having known her as a child I looked still with the child's view of immortality. I thought that she would always be there and that we could pick up our friendship where we had left off.

As doctors we should understand better than others the lottery of life and death. The news of her death brought home to me that friendship needs to be nurtured and cultivated, and that an old friend is the most precious of all. I feel cheated and angry that she did not tell me what was happening to her and give me the opportunity to see her. Most of all I feel angry with myself for not finding the time and making the effort to visit and catch up with my oldest friend before her funeral.

For Cicely Mary Haldane Tanous (née Breese) who died on 28 July 1995 (p 1224).-CATHERINE GREBENIK is a consultant anaesthetist in Oxford 